\title{
Tobacco smoker identity
}

Addiction Ontology

\section{Source}

Addiction Ontology

Definition: A self identity in which a person represents themselves as a tobacco smoker.

Comment: Identity in this case is the application of a label to oneself. The mental representation that this label references may be different in different people.

This definition was imported from the Addiction Ontology (see https://addictovocab.org/). Comments and suggestions for improvements are welcome using the Qeios review system.

Definitions imported from the Addiction Ontology (AddictO) are what are known as 'ontological definitions'. See this article in Qeios for an explanation https://www.qeios.com/read/YGIF9B.

Ontological definitions can sometimes be hard to read. In those cases we also include an informal definition.

Definitions also often require elaboration to make it clear how they should be used and what they include. In those cases we include a comment.

Also, definitions sometimes require an explanation as to how they came about to help users understand how they relate to alternative definitions. In those cases we include a curator note. 